\title{
INDECOMPOSABLE MODULES OVER NAGATA VALUATION DOMAINS
}

\author{
D. ARNOLD AND M. DUGAS
}

(Communicated by Ronald M. Solomon)

\begin{abstract}
For a discrete valuation ring $R$, let $\mathrm{fr}(R)$ be the supremum of the ranks of indecomposable finite rank torsion-free $R$-modules. Then $\operatorname{fr}(R)=$ $1,2,3$, or $\infty$. A complete list of indecomposables is given if $\operatorname{fr}(R) \leq 3$, in which case $R$ is known to be a Nagata valuation domain.
\end{abstract}

Let $R$ be a discrete valuation ring with prime $p$ and quotient field $Q$, and let $R^{*}$ be the $p$-adic completion of $R$ with quotient field $Q^{*}$. Define $\operatorname{fr}(R)=\sup \{\operatorname{rank} X: X$ indecomposable torsion-free $R$-module of finite rank $\}$. In this paper, we show that $\operatorname{fr}(R)=1,2,3$, or $\infty$. This resolves a conjecture by $\mathrm{P}$. $\mathrm{Va}^{\prime}$ mos that $\operatorname{fr}(R)=1,2$, or $\infty$.

It is well known that $\operatorname{fr}(R)=\infty$ in case $\left[Q^{*}: Q\right]$ is infinite and $\operatorname{fr}(R)=1$ if $\left[Q^{*}: Q\right]=1$. Call $R$ a Nagata valuation domain if $2 \leq\left[Q^{*}: Q\right]$ is finite [Z]. In this case char $Q^{*}=q>0 ; Q^{*}=Q(u)$ for some unit $u$ of $R^{*}$ with $u^{n}=\lambda$, a unit of $R$; and $\left[Q^{*}: Q\right]$ is a power of $q[\mathrm{~V}, \mathrm{R}]$. Examples of Nagata valuation domains are given in $[\mathrm{N}]$ and $[\mathrm{V}]$.

Zanardo $[Z]$ shows that if $\left[Q^{*}: Q\right]=2$, then $\operatorname{fr}(R)=2$. Moreover, in this case there are, up to isomorphism, only three indecomposables: $R, Q$, and $R^{*}$. His example showing that $\operatorname{fr}(R) \geq 6$ for $\left[Q^{*}: Q\right]=3$ is in error.

Henceforth, assume $\left[Q^{*}: Q\right]=n \geq 2$. Then $Q^{*}$ is a splitting field for each finite rank $R$-module $X$; i.e., $R^{*} \otimes X$ is the direct sum of a free $R^{*}$-module and a $Q^{*}$-module. Thus, quasi-homomorphism results of Lady [L1, L3] for modules over a discrete valuation ring with a fixed splitting field are applicable.

As summarized in [L1, Theorem 1] and proved in [L3, Theorem 5.1], for:

$n=2$, there are three strongly indecomposables: $R, Q$, and $R^{*}$;

$n=3$, there are five strongly indecomposables: $R, Q, R^{*}, C^{-} R$ (p-rank 1 , rank 2), and $C^{+} R^{*} \quad(p$-rank 2, rank 3 );

$n=4$, there are strongly indecomposables of arbitrarily large finite rank, but all strongly indecomposables are potentially describable (tame representation type);

$n \geq 5$, there are strongly indecomposables of arbitrarily large finite rank, but a description is generally regarded as hopeless (wild representation type).

Received by the editors February 25, 1993.

1991 Mathematics Subject Classification. Primary 13C05, 13E05.

Research supported in part by NSF grant DMS- 9101000 . 
Since strongly indecomposables are indecomposable, Lady's theorem yields $\operatorname{fr}(R)=\infty$ for $n \geq 4$. We give an alternate proof by easily constructed examples in $\S 3$. This is sufficient for our purposes and avoids the deep arguments used in [L3].

The only unresolved case is $n=3$. In this case, we show that $\operatorname{fr}(R)=3$ and give a complete list of indecomposables up to isomorphism: $R, Q, R^{*}, C^{-} R$, and infinitely many of $p$-rank 2 , rank 3 (all quasi-isomorphic to $C^{+} R^{*}$ ). The strongly indecomposable $R$-module $C^{+} R^{*}$ is the quasi-homomorphism dual of $R^{*}$ defined in [A1].

\section{Preliminaries}

The $p$-rank of an $R$-module $X$ is the $R / p R$-dimension of $X / p X$. Fundamental properties of $p$-rank are given in [A1].

Lemma 1.1 [A1, Proposition 1.3, Lemma 1.5]. Two finite rank $R$-modules $G$ and $H$ are quasi-isomorphic if and only if $p$ - $\operatorname{rank} G=p-\operatorname{rank} H, \operatorname{rank} G=$ rank $H$, and there is a monomorphism $f: G \rightarrow H$. Moreover, quasi-isomorphism implies isomorphism for modules of p-rank 1 .

\section{INDECOMPOSABLES FOR $\left[Q^{*}: Q\right]=3$}

As noted in the introduction, $\operatorname{char} Q=3$ and $Q^{*}=Q(u)$ for some unit $u$ of $R^{*}$ with $u^{3}=\lambda$, a unit of $R$. This notation is maintained throughout the rest of this section.

Define $A[u]$ to be the pure $R$-submodule of $R^{*}$ generated by $\{1, u\}$. Then $A[u]=(Q \oplus Q u) \cap R^{*}$ is strongly indecomposable with $p$-rank 1 and rank 2 and, hence, is quasi-isomorphic to $C^{-} R$ by Lady's theorem. The following lemma is proved in [Z, Proposition 5] using Kurosch matrix-invariant arguments from [A1]. However, it can also be proved directly from the definition of $A[u]$ (a proof is not included).

Lemma $2.1[\mathrm{Z}$, Corollary 12, Theorem 8]. The module $A[u]$ is (strongly) indecomposable. Moreover, if $X$ is an indecomposable $R$-module of rank $\leq 2$, then $X$ is isomorphic to $R, Q$, or $A[u]$.

Next let $a, b \in R^{*} \backslash R$ and define $A[a, b]$ to be the pure $R$-submodule of $R^{*} \oplus R^{*}$ generated by $(1,0),(0,1)$, and $(a, b)$. In particular, $Q A[a, b]=$ $Q(1,0) \oplus Q(0,1) \oplus Q(a, b)$ and $A[a, b]=Q A[a, b] \cap\left(R^{*} \oplus R^{*}\right)$. Up to isomorphism, this definition of $A[a, b]$ coincides with that of $[Z]$. Then $A[a, b]$ has $p$-rank 2 and rank 3. A routine argument shows that $A[a, b]$ is (strongly) indecomposable if and only if $\{1, a, b\}$ is a $Q$-independent set. In this case, $A[a, b]$ is quasi-isomorphic to $C^{+} R^{*}$ by Lady's theorem. Moreover, $A\left[u, u^{2}\right]$ is the quasi-homomorphism dual of $R^{*}$, noting that $R^{*}$ has $p$-rank 1 and rank 3.

Lemma 2.2. Suppose that $(a, b)$ and $(c, d)$ are $R^{*}$-vectors.

(a) If $(c, d)=s(a, b) M+P$ for an invertible $2 \times 2 R$-matrix $M, a Q$ vector $P$, and $0 \neq s \in Q$, then $A[a, b] \approx A[c, d]$.

(b) $A\left[u, p^{i} u^{2}\right] \approx A\left[p^{i} u, u^{2}\right]$.

(c) If $r$ is a unit of $R$ and $j>i$, then $A\left[u+p^{i} r u^{2}, p^{j} u^{2}\right] \approx A\left[u, p^{j} u^{2}\right]$. 
Proof. (a) Define an $R^{*}$-automorphism $\phi$ of $R^{*} \oplus R^{*}$ by $\phi(x)=x M^{-1}$. Then $\phi$ induces a homomorphism $A[c, d] \rightarrow A[a, b]$ since $(Q \oplus Q) M^{-1}$ is contained in $Q \oplus Q$ and $(c, d) M^{-1}=s(a, b)+P M^{-1}$. In fact, this is an isomorphism since $A[a, b]$ and $\phi(A[c, d])$ are both pure rank-3 submodules of $R^{*} \oplus R^{*}$.

(b) Let $A=A\left[u, p^{i} u^{2}\right]$ and $B=A\left[p^{i} u, u^{2}\right]$ with $i \geq 1$. There is an $R^{*}$-endomorphism $\phi$ of $R^{*} \oplus R^{*}$ defined by

$$
\begin{aligned}
& \phi(1,0)=(1,1)=(1,0)+(0,1) \in A, \\
& \phi(0,1)=\left(-u^{-2},-p^{i} u^{-1}+p^{2 i}\right)=-\lambda^{-1}\left(u, p^{i} u^{2}\right)+p^{2 i}(0,1) \in A,
\end{aligned}
$$

recalling that $u^{3}=\lambda$. Now $\phi$ is an automorphism as

$$
\left(\begin{array}{cc}
1 & 1 \\
-u^{-2} & -p^{i} u^{-1}+p^{2 i}
\end{array}\right)
$$

has determinant $d \equiv-u^{-2}\left(\bmod p R^{*}\right)$, a unit of $R^{*}$. Moreover, $\phi(B)$ is contained in $A$ since

$$
\begin{aligned}
\phi\left(p^{i} u, u^{2}\right) & =p^{i} u(1,1)+u^{2}\left(-u^{-2},-p^{i} u^{-1}+p^{2 i}\right)=\left(p^{i} u-1, p^{2 i} u^{2}\right) \\
& =p^{i}\left(u, p^{i} u^{2}\right)-(1,0) \in A .
\end{aligned}
$$

It follows that $\phi: B \rightarrow A$ is an isomorphism.

(c) Let $A=A\left[u, p^{j} u^{2}\right]$ and $B=A\left[u+p^{i} r u^{2}, p^{j} u^{2}\right]$, and assume that either $i \geq 1$ or else $i=0$ and $r u$ is not congruent to 1 modulo $p R^{*}$.

Define an $R^{*}$-endomorphism $\phi$ of $R^{*} \oplus R^{*}$ by

$$
\begin{aligned}
\phi(1,0) & =\left(1-p^{i} r u,-p^{i+j} r u^{2}+p^{2 i+j} r^{2} \lambda\right) \\
& =(1,0)-p^{i} r\left(u, p^{j} u^{2}\right)+p^{2 i+j} r^{2} \lambda(0,1) \in A, \\
\phi(0,1) & =\left(0,1-p^{3 i} r^{3} \lambda\right)=\left(1-p^{3 i} r^{3} \lambda\right)(0,1) \in A .
\end{aligned}
$$

Then $\phi$ is an automorphism if $i \geq 1$, since the coefficient determinant $d=$ $\left(1-p^{i} r u\right)\left(1-p^{3 i} r^{3} \lambda\right) \equiv 1\left(\bmod p R^{*}\right)$. If $i=0$, then $d=(1-r u)\left(1-\lambda r^{3}\right)$. Since char $Q^{*}=3,1-\lambda r^{3}=1-(r u)^{3}=(1-r u)^{3}$, whence $d=(1-r u)^{4}$. Thus, $\phi$ is an automorphism, as $r u$ is not congruent to $1 \bmod p R^{*}$.

Now $\phi(B)$ is contained in $A$ since

$$
\begin{aligned}
\phi(u & \left.+p^{i} r u^{2}, p^{j} u^{2}\right) \\
& =\left(u+p^{i} r u^{2}\right) \phi(1,0)+p^{j} u^{2} \phi(0,1) \\
& =\left(u+p^{i} r u^{2}\right)\left(1-p^{i} r u,-p^{i+j} r u^{2}+p^{2 i+j} r^{2} \lambda\right)+p^{j} u^{2}\left(0,1-p^{3 i} r^{3} \lambda\right) \\
& =\left(u-p^{2 i} r^{2} \lambda, p^{j} u^{2}-p^{i+j} r \lambda\right) \\
& =\left(u, p^{j} u^{2}\right)-p^{2 i} r^{2} \lambda(1,0)-p^{i+j} r \lambda(0,1) \in A,
\end{aligned}
$$

recalling that $u^{3}=\lambda$. As in the proof of $(b), B \approx \phi(B)=A$.

It remains to show that it is sufficient to assume that either $i \geq 1$ or else $i=0$ and $u$ is not congruent to 1 modulo $p R^{*}$. To see this, assume that $i=0$ and $r u=1+p s$ for some $s=s_{0}+s_{1} u+s_{2} u^{2} \in R^{*}$. Then $u+r u^{2}=$ $\left(2+p s_{0}\right) u+p s_{1} u^{2}+p s_{2} \lambda$. Since $p s_{2} \lambda \in Q$, it follows from (a) that $B=$ $A\left[u+r u^{2}, p^{j} u^{2}\right] \approx A\left[\left(2+p s_{0}\right) u+p s_{1} u^{2}, p^{j} u^{2}\right]$. As char $Q=3,2+p s_{0}=$ $-1+p s_{0}$ is a unit of $R^{*}$. Thus, $B \approx A\left[u+p t u^{2}, p^{j} u^{2}\right]$ for $t=\left(2+p s_{0}\right)^{-1} s_{1}$ by (a). If $i^{\prime}=p$-height $(p t) \geq j$, an application of (a) shows that $B \approx A$. Otherwise, $j<i^{\prime}$ and $i^{\prime} \geq 1$, as desired. 
Theorem 2.3. If $X$ is an indecomposable R-module of rank 3, then $X$ is isomorphic to $R^{*}$ or $A\left[u, p^{j} u^{2}\right]$ for some $j$.

Proof. Note that $p$-rank $X \neq 0$ or 3 , as $X$ is reduced with no free summands (see [A1]). If $p$-rank $X=1$, then $X$ embeds in its completion which is isomorphic to $R^{*}$. Since $R^{*}$ also has $p$-rank 1 and rank $3, X \approx R^{*}$ by Lemma 1.1 .

Now assume that $X$ is indecomposable with $p$-rank 2 and rank 3. Then $X \approx$ $A[a, b]$ with $(a, b)=\left(u, u^{2}\right) M$ for some $2 \times 2 R$-matrix $M$ with $\operatorname{det} M \neq$ 0 [Z]. We outline another proof that avoids matrix invariants. Let $R x \oplus R y$ be a basic submodule of $X$ and extend to a maximal free submodule $R x \oplus R y$ $\oplus R z$ of $X$. Then $X$ embeds as a pure submodule of $R^{*} x \oplus R^{*} y \approx\left(R^{*} \otimes X\right) /$ $d\left(R^{*} \otimes X\right)$, where $d\left(R^{*} \otimes X\right)$ is the maximal divisible submodule. It follows that $X \approx A[a, b]$, where image $z=a x \oplus b y$ for $a, b \in R^{*}$. Since $Q^{*}=Q(u)=$ $Q \oplus Q u \oplus Q u^{2}$, we may write $(a, b)=\left(u, u^{2}\right) M+P$ for some $R$-matrix $M$ and $R$-vector $P$. Apply Lemma 2.2(a) to see that, up to isomorphism, $P$ may be chosen to be 0 .

In view of Lemma 2.2(a), the isomorphism class of $A[a, b]$ is preserved by invertible $R$-column operations on $M$. In particular, it suffices to assume that $M$ is of the form

$$
\left(\begin{array}{cc}
p^{k} & 0 \\
p^{r} & p^{j}
\end{array}\right)
$$

with $i<j$ and $r$ either 0 or a unit of $R$. This follows from the observation that if an element in a row has least $p$-height, then the other entry in its row can be set to 0 using an invertible $R$-column operation. Moreover, column interchange and multiplication of a column by a unit are invertible $R$-operations.

We now have $X \approx A[a, b]$ with $(a, b)=\left(p^{k} u+p^{i} r u^{2}, p^{j} u^{2}\right), j>i$ and $r$ either 0 or a unit of $R$.

First, assume $k \leq i$. Then $X \approx A\left[u+p^{i-k} r u^{2}, p^{j-k} u^{2}\right]$ by Lemma 2.2(a). Moreover, $A\left[u+p^{i-k} r u^{2}, p^{j-k} u^{2}\right] \approx A\left[u, p^{j-k} u^{2}\right]$ via Lemma 2.2(c). Thus, $X \approx A\left[u, p^{j-k} u^{2}\right]$.

Now assume $k>i$. Factor out $p^{i}$ and apply Lemma 2.2(a) to assume, up to isomorphism, that $[a, b]=\left[p^{k-i} u+r u^{2}, p^{j-i} u^{2}\right]$. If $r=0$, then $X \approx$ $A[a, b] \approx A\left[u, p^{t} u^{2}\right]$ for some $t$, obtained by factoring out $p^{\min \{k-i, j-i\}}$ and applying Lemma $2.2(\mathrm{~b})$ in the case $k-i>j-i$.

Finally assume that $r$ is a unit. Then $X \approx A[a, b]=A\left[r u^{2}+p^{k-i} u, p^{j-i} u^{2}\right]$ $\approx A\left[u^{2}+p^{i^{\prime}} r^{\prime} u, p^{j^{\prime}} u^{2}\right]$ for $i^{\prime}=k-i, j^{\prime}=j-i$, and $r^{\prime}=r^{-1}$ (Lemma 2.2(a)). Since $\left(u^{2}\right)^{2}=u \lambda$, substituting $v$ for $u^{2}$ in the latter term and relabeling exponents and units gives $X \approx A\left[v+p^{i} r v^{2}, p^{j} v\right]$ for a unit $r=r^{\prime} / \lambda$ of $R$. Invertible $R$-column operations on

$$
\left(\begin{array}{cc}
1 & p^{j} \\
p^{i} r & 0
\end{array}\right)
$$

reduce to the case that $X \approx A\left(v+p^{i} r v^{2}, p^{i+j} v^{2}\right)$. However, $Q^{*}=Q(u)=Q(v)$ with $v^{3}=\lambda^{2}$, a unit of $R$. Thus, Lemma 2.2, with $u$ replaced by $v$, is true. The argument of the first case then shows that $X \approx A\left[v, p^{t} v^{2}\right]$ for some $t$. Hence, by Lemma $2.2, X \approx A\left[u^{2}, p^{t} \lambda u\right] \approx A\left[u^{2}, p^{t} u\right] \approx A\left[p^{t} u, u^{2}\right] \approx$ $A\left[u, p^{t} u^{2}\right]$, as desired.

For finite rank torsion-free $R$-modules $G$ and $H$, define $S_{G}(H)$ to be the 
image of the evaluation map $\operatorname{Hom}(G, H) \otimes_{R} G \rightarrow H$. Fundamental properties of $S_{G}(-)$ are given in [A2, Chapter 5] for torsion-free abelian groups of finite rank.

Proposition 2.4. (a) If $A\left[u, p^{j} u^{2}\right] \approx A\left[u, p^{j} u^{2}\right]$, then $i=j$.

(b) There are embeddings $A\left[u, p^{i} u^{2}\right] \rightarrow A\left[u, p^{i-1} u^{2}\right]$ and $A\left[u, p^{i-1} u^{2}\right] \rightarrow$ $A\left[u, p^{i} u^{2}\right]$. In each case the image has index $p$. $H$.

(c) If $G$ and $H$ are indecomposable with p-rank 2 and rank 3 , then $S_{G}(H)=$

Proof. (a) can be proven as in [Z, Proposition 16] for the case $i=0, j=1$. We outline an alternate proof that avoids matrix invariants. An $R$-isomorphism $\phi: A=A\left[u, p^{i} u^{2}\right] \rightarrow B=A\left[u, p^{j} u^{2}\right]$ lifts to an $R^{*}$-isomorphism of completions $\phi^{*}: A^{*}=R^{*} \oplus R^{*} \rightarrow B^{*}=R^{*} \oplus R^{*}$. Since $\phi\left(u, p^{i} u^{2}\right) \in B$ and $\phi^{-1}\left(u, p^{j} u^{2}\right) \in A$, it follows from a computation of $p$-heights that $i=j$.

(b) There is a monomorphism $f: A\left[u, p^{i-1} u^{2}\right] \rightarrow A\left[u, p^{i} u^{2}\right]$ induced by an $R^{*}$-endomorphism $\phi$ of $R^{*} \oplus R^{*}$ with $\phi(1,0)=(1,0)$ and $\phi(0,1)=(0, p)$. Moreover, there is a monomorphism $f^{\prime}: A\left[u, p^{i} u^{2}\right] \rightarrow A\left[u, p^{i-1} u^{2}\right]$ induced by $\phi^{\prime}(1,0)=(p, 0)$ and $\phi^{\prime}(0,1)=(0,1)$. Note that $f f^{\prime}=p$ and $f^{\prime} f=p$. Hence, if $H_{i}=A\left[u, p^{i} u^{2}\right]$, then $p H_{i}$ is contained in image $f$. But $p$-rank $H_{i}=2$ and $H_{i}$ is not isomorphic to $H_{i-1}$ by (b). It follows that $H_{i}$ /image $f \approx$ $R / p R$. Similarly, $H_{i-1} /$ image $f^{\prime} \approx R / p R$.

(c) For $i \geq 1$ and for $\phi^{\prime}$ and $\phi$ defined as in the proof of (b), there is $g$ : $A\left[p^{i-1} u, u^{2}\right] \rightarrow A\left[p^{i} u, u^{2}\right]$ induced by $\phi^{\prime}$ and $g^{\prime}: A\left[p^{i} u, u^{2}\right] \rightarrow A\left[p^{i-1} u, u^{2}\right]$ induced by $\phi$ with $g g^{\prime}=p$ and $g^{\prime} g=p$. It now follows that if $G_{i}=$ $A\left[p^{i} u, u^{2}\right]$, then $G_{i} /$ image $g \approx R / p R \approx G_{i-1} /$ image $g^{\prime}$.

In view of Theorem 2.3 , it is sufficient to show that

$$
f \oplus \delta_{i} g \delta_{i-1}^{-1}: H_{i-1} \oplus H_{i-1} \rightarrow H_{i} \quad \text { and } \quad f^{\prime} \oplus \delta_{i-1} g^{\prime} \delta_{i}^{-1}: H_{i} \oplus H_{i} \rightarrow H_{i-1}
$$

are onto, for $\delta_{i}$ the isomorphism $G_{i}=A\left[p^{i} u, u^{2}\right] \rightarrow A\left[u, p^{i} u^{2}\right]=H_{i}$ given in Lemma 2.2(b). Assume that $f \oplus \delta_{i} g \delta_{i-1}^{-1}$ is not onto. Since $H_{i} / p H_{i} \approx R / p R \oplus$ $R / p R$ and $p H_{i}$ is properly contained in both the image of $f$ and the image of $\delta_{i} g \delta_{i-1}^{-1}$, it follows that image $f=$ image $\delta_{i} g \delta_{i-1}^{-1}$. Hence, $f \delta_{i-1}\left(G_{i-1}\right)=$ $\delta_{i} g\left(G_{i-1}\right)$. But this is a contradiction, as can be seen by observing that $f$ is a restriction of $\phi$ and $g$ is a restriction of $\phi^{\prime}$. The proof that $f^{\prime} \oplus \delta^{-1} g^{\prime}$ is onto is analogous.

Lemma 2.5. Assume that $X$ is a finite rank $R$-module with submodule $K$ such that $A=X / K \approx A[u]$ or $A\left[u, p^{i} u^{2}\right]$ for some $i \geq 0$. If $S_{A}(X)=X$, then $K$ is a summand of $X$.

Proof. It suffices to prove that $\operatorname{End}(A[u])$ and $\operatorname{End}\left(A\left[u, p^{i} u^{2}\right]\right)$ are commutative. This is a consequence of [AR2, Theorems 5.6 and 5.8] as the abelian group proof therein carries over to modules over discrete valuation rings. Recall that $A[u]$ has $p$-rank 1 and is reduced. Hence its completion is isomorphic to $R^{*}$. In particular, $\operatorname{End}(A[u])$ is isomorphic to a subring of $R^{*}$. Moreover, $A\left[u, p^{i} u^{2}\right]$ is quasi-isomorphic to $A\left[u, u^{2}\right]$ which is the dual of $R^{*}$, as noted above. Thus, $Q \operatorname{End}\left(A\left[u, p^{i} u^{2}\right]\right)=Q \operatorname{End}\left(A\left[u, u^{2}\right]\right)=Q \operatorname{End}\left(R^{*}\right)=Q R^{*}$. It follows that $\operatorname{End}\left(A\left[u, p^{i} u^{2}\right]\right)$ is commutative.

Theorem 2.6. If $X$ is a finite rank $R$-module, then $X$ is the direct sum of modules of rank $\leq 3$. 
Proof. Choose pure strongly indecomposable submodules $X_{i}$ of $X$ with $X /\left(X_{1} \oplus \cdots \oplus X_{m}\right) p^{k}$-bounded. Each $X_{j}$ is isomorphic to $R, R^{*}, Q, A[u]$, or $A\left[u, p^{r} u^{2}\right]$ for some $r \geq 0$ by Lady's theorem, Lemma 2.1, and Theorem 2.3. If $X_{i}$ is isomorphic to the pure injective module $R^{*}$ or $Q$, then $X_{i}$ is a summand of $X$. Moreover, if $X_{i} \approx R$, then $X$ has a cyclic summand, since $X$ modulo the pure submodule generated by $\left\{X_{j}: j \neq i\right\}$ is isomorphic to $R$.

We may now assume that each $X_{j}$ is isomorphic to $A[u]$ of some $A\left[u, p^{i} u^{2}\right]$. By induction on $\operatorname{rank} X$ and $\left|X /\left(X_{1} \oplus \cdots \oplus X_{m}\right)\right|$, it suffices to further assume that $X /\left(X_{1} \oplus \cdots \oplus X_{m}\right) \approx R / p R$ and prove that $X$ has a summand of rank $\leq 3$. Write $X=\left(X_{1} \oplus \cdots \oplus X_{m}\right)+R\left(x_{1}+\cdots+x_{m}\right) / p$. Let $K$ be the pure submodule of $X$ generated by $\left\{X_{j}: j \neq 1\right\}$ and $A=X / K$, quasi-isomorphic to $X_{1}$. Then $A$ has $p$-rank 1, rank 2 or $p$-rank 2 , rank 3 and has no free summands, being quasi-isomorphic to a strongly indecomposable $X_{1}$. Hence, $A$ is indecomposable [A1, Proposition 4.1].

It is now sufficient to prove that $S_{A}(X)=X$, in which case $X$ has a summand isomorphic to $A$ of rank $\leq 3$ by Lemma 2.5. There is some $Y=X_{i}$, say $i=1$, with $S_{Y}\left(X_{j}\right)=X_{j}$ for each $j$. This follows from the natural exact sequence $A\left[u, p^{r} u^{2}\right] \rightarrow A[u] \rightarrow 0$, Proposition 2.4(c), and the assumption that each $X_{j} \approx A[u]$ or $A\left[u, p^{r} u^{2}\right]$. Moreover, for $A=X / K \approx X_{1}+R\left(x_{1} / p\right)$, $S_{A}\left(X_{j}\right)=X_{j}$ for each $j$, again by Proposition 2.4(c) or Lemma 2.1 and the fact that $A$ is indecomposable.

Write $X_{i}^{\prime}=p X_{i}+R x_{i}$, an indecomposable module for the same reason that $A$ is. For each $i$, there is $y_{i} \in A$, a unit $r_{i}$ of $R$, and $f_{i}: A \rightarrow X_{i}^{\prime}$ with $f_{i}\left(y_{i}\right) \equiv r_{i} x_{i}\left(\bmod p X_{i}\right)$. This is because if $X_{i}^{\prime}=S_{A}\left(X_{i}^{\prime}\right)$ is contained in $p X_{i}$, then $x_{i} \in p X_{i}$ and letting $r_{i}=1$ will do. Note, for future reference, that we may as well assume that $f_{i}\left(y_{i}\right) \equiv x_{i}\left(\bmod p X_{i}\right)$. To see this, choose a unit $s_{i}$ of $R$ with $1=r_{i} s_{i}+p t_{i}, t_{i} \in R$. Then $s_{i} f_{i}\left(y_{i}\right) \equiv x_{i}\left(\bmod p X_{i}\right)$, as desired.

We begin with the case $m=2$ and find $x \in A$ and $g_{i}: A \rightarrow X_{i}^{\prime}$ with $g_{1}(x) \equiv$ $x_{1}\left(\bmod p X_{1}\right)$ and $g_{2}(x) \equiv x_{2}\left(\bmod p X_{2}\right)$. If either $f_{1}\left(y_{2}\right) \equiv s_{1} x_{1}\left(\bmod p X_{1}\right)$ or $f_{2}\left(y_{1}\right) \equiv s_{2} x_{2}\left(\bmod p X_{2}\right)$ for units $s_{1}, s_{2}$ of $R$, then let $x=y_{2}$, respectively, $x=y_{1}$. Otherwise, $f_{1}\left(y_{2}\right) \in p X_{1}$ and $f_{2}\left(y_{1}\right) \in p X_{2}$. In this case, let $x=$ $y_{1}+y_{2}$. In any case, there are units $t_{i}$ of $R$ with $f_{i}(x) \equiv t_{i} x_{i}\left(\bmod p X_{i}\right)$. As above, choose $g_{i}$ to be an appropriate $R$-unit multiple of $f_{i}$.

Next let $A^{\prime}=p A+R x$, an indecomposable submodule of $A$ for the same reason that $A$ is indecomposable. Restriction induces a well-defined $\phi=g_{1} \oplus$ $g_{2}: A^{\prime} \rightarrow p X=p X_{1} \oplus p X_{2}+R\left(x_{1} \oplus x_{2}\right)$ with $\phi(x) \in\left(x_{1} \oplus x_{2}\right)+p X_{1} \oplus p X_{2}$. Since $S_{A}\left(A^{\prime}\right)=A^{\prime}$ by Proposition 2.4(c) and $S_{A}\left(X_{i}\right)=X_{i}$ for each $i$, it follows that $S_{A}(p X)=p X$ and so $S_{A}(X)=X$. This completes the proof for $m=2$.

We illustrate an induction argument with $m=3$. From the $m=2$ case $S_{A}\left(X_{12^{\prime}}\right)=X_{12^{\prime}}$ for $X_{12^{\prime}}=p X_{1} \oplus p X_{2}+R\left(x_{1} \oplus x_{2}\right)$. Consequently, there is $x \in A$ and $g_{12}: A \rightarrow X_{12^{\prime}}$ with $g_{12}(x) \equiv\left(x_{1} \oplus x_{2}\right)\left(\bmod p X_{1} \oplus p X_{2}\right)$. Otherwise, $x_{1} \oplus x_{2} \in S_{A}\left(X_{12^{\prime}}\right)=X_{12^{\prime}}$ is contained in $p X_{1} \oplus p X_{2}$. Recall that there is $y_{3}=A$ and $f_{3}: A \rightarrow X_{i}^{\prime}$ with $f_{3}\left(y_{3}\right) \equiv x_{3}\left(\bmod p X_{3}\right)$. If $f_{3}(x) \equiv s_{3} x_{3}\left(\bmod p X_{3}\right)$ for some unit $s_{3}$ of $R$, then let $a=x$. If $g_{12}\left(y_{3}\right) \equiv s\left(x_{1} \oplus x_{2}\right)\left(\bmod p X_{1} \oplus p X_{2}\right)$ for some unit $s$ of $R$, then let $a=y_{3}$. Otherwise, let $a=x+y_{3}$. It follows that $a \in A$ with $g_{12}(a) \equiv r\left(x_{1} \oplus x_{2}\right)\left(\bmod p X_{1} \oplus p X_{2}\right)$ and $f_{3}(a) \equiv r_{3} x_{3}\left(\bmod p X_{3}\right)$ for units $r$ and $r_{3}$ of $R$. As in the $m=2$ case, we may assume that $r=$ $r_{3}=1$ and construct $\phi: A^{\prime}=p A+R a \rightarrow p X$ with $\phi(a) \equiv\left(x_{1} \oplus x_{2} \oplus x_{3}\right)$ 
$\left(\bmod p X_{1} \oplus p X_{2} \oplus p X_{3}\right)$. It follows, as above, that $S_{A}(X)=X$.

The proof is concluded by an induction on $m$; the argument for passing from $m$ to $m+1$ is analogous to that of the preceding paragraph.

\section{INDECOMPOSABLES FOR $\left[Q^{*}: Q\right]=n \geq 4$}

The following are examples showing that $\operatorname{fr}(R)=\infty$ for $\left[Q^{*}: Q\right]=n \geq$ 4. The detailed computations needed to verify that the modules are actually strongly indecomposable are omitted.

Example 3.1. Assume $n \geq 4$. Given $m \geq 2$, there is a strongly indecomposable $R$-module with $p$-rank $m$ and rank $2 m$.

Proof. Case I: char $Q^{*} \geq 5$. Since $Q^{*}$ is purely inseparable over $Q$, there is $u \in R^{*}$ such that $1, u, u^{2}, u^{3}$, and $u^{4}$ are $Q$-independent. Let $M$ be an $m \times m$ simple Jordan block $R$-matrix, i.e., the diagonal elements of $M$ are a fixed unit $\lambda$ of $R$, the super diagonal elements are all 1's, and the remaining entries are 0 . Define $X=A[\Gamma]=\left(R^{*}\right)^{m} \cap\left(Q^{m} \oplus Q^{m} \Gamma\right)$, where $\Gamma=u M+u^{2} I_{m}$, and $R$-module with $p$-rank $m$ and rank $2 m$.

It can be shown that $\operatorname{End}(X)$ is represented by the set of $2 m \times 2 m R$ matrices $\left(\begin{array}{cc}\Pi & 0 \\ 0 & \Pi\end{array}\right)$ with $\Pi M=M \Pi$. This can be seen by equating $Q$-coefficients $1, u, u^{2}, u^{3}$, and $u^{4}$. Consequently, $Q \operatorname{End}(X) \approx Q[M] \approx Q[x] /\left\langle(x-\lambda)^{m}\right\rangle$ is a ring with no nontrivial idempotents, whence $X$ is strongly indecomposable.

Case II: $\operatorname{char} Q^{*}=3$. If there is $u \in R^{*}$ with $1, u, u^{2}, u^{3}$, and $u^{4} Q$ independent, the construction of Case I suffices. Otherwise, there are $u, v \in R^{*}$ with $u^{3}, v^{3} \in R$ and $1, u, v, u^{2} v, v^{2} u, u^{2} v^{2}, u^{2}$, and $v^{2}$ are $Q$-independent. Choose $M$ as in Case $I$, and define $X=A[\Gamma]$ for $\Gamma=u M+v I$. An argument similar to that of Case I shows that $Q \operatorname{End}(X) \approx Q[X] /\left\langle(x-\lambda)^{m}\right\rangle$ and $X$ is strongly indecomposable.

Case III: char $Q^{*}=2$. We are left with two possibilities not covered in Case I: there is $u \in R^{*}$ with $1, u, u^{2}$, and $u^{3} Q$-independent and $u^{4} \in R$, or there is $u, v \in R^{*}$ with $u^{2}, v^{2} \in R$ and $\{1, u, v, u v\} Q$-independent. In the first case, define $X=A[\Gamma]$ for $\Gamma=u M+u^{3} I$. An argument similar to that of Case I shows that $X$ is strongly indecomposable.

For the second case, define $X=A[\Gamma]$ for $\Gamma=u M+v I$. Once again, it can be shown that $Q \operatorname{End}(X)$ has no nontrivial idempotents, but the argument is slightly more complicated than the previous cases.

\section{ACKNOWLEDGMENT}

We thank the referee for helpful suggestions that improved the readability of the paper. Undefined notation and terminology is as in [A1], [A2], or [F].

\section{REFERENCES}

[A1] D. Arnold, A duality for torsion-free modules of finite rank over a discrete valuation ring, Proc. London Math. Soc. (3) 24 (1972), 204-216.

[A2] __, Finite rank torsion free abelian groups and rings, Lecture Notes in Math., vol. 931, Springer-Verlag, New York, 1982.

[F] L. Fuchs, Infinite Abelian groups, Vols. I and II, Academic Press, New York, 1970 and 1973.

[L1] L. Lady, Abelian group theory, Lecture Notes in Math., vol. 616, Springer-Verlag, New York, 1977, pp. 168-172. 
[L2] _ A seminar on splitting rings for torsion-free modules over Dedekind domains, Abelian Group Theory, Lecture Notes in Math., vol. 1006, Springer-Verlag, New York, 1983, pp. $1-48$.

[L3] _ Splitting fields for torsion-free modules over Dedekind domains, J. Algebra 49 (1977), 261-275.

[N] M. Nagata, Local rings, Wiley Interscience, New York, 1962.

[R] P. Ribenboim, On the completion of a valuation ring, Math. Ann. 155 (1964), 392-396.

[V] $\mathbf{P}$. Va'mos, Decomposition problems for modules over valuation domains, J. London Math. Soc. 41 (1990), 10-26.

[Z] P. Zanardo, Kurosch invariants for torsion-free modules over Nagata valuation domains, J. Pure Appl. Algebra 82 (1992), 195-209.

Department of Mathematics, Baylor University, Waco, TeXas 76798-7328

E-mail address: arnolddebaylor .edu

E-mail address: dugasmebaylor.edu 